\title{
Interplay between charge-lattice interaction and strong electron correlations in cuprates: phonon anomaly and spectral kinks
}

\author{
G. De Filippis ${ }^{1}$, V. Cataudella ${ }^{1}$, R. Citro ${ }^{2}$, C.A. Perroni ${ }^{1}$, A. S. Mishchenko ${ }^{3,4}$ and N. Nagaosa ${ }^{3,5}$ \\ ${ }^{1}$ SPIN-CNR and Dip. di Scienze Fisiche - Università di Napoli Federico II - I-80126 Napoli, Italy \\ ${ }^{2}$ CNISM and Dip. di Fisica "E. R. Caianiello"- Università di Salerno, I-84100 Salerno, Italy \\ ${ }^{3}$ Cross-Correlated Materials Research Group (CMRG), ASI, RIKEN, Wako 351-0198, Japan \\ ${ }^{4} R R C$ "Kurchatov Institute" - 123182 - Moscow - Russia \\ ${ }^{5}$ Department of Applied Physics, The University of Tokyo, 7-3-1 Hongo, Bunkyo-ku, Tokyo 113, Japan
}

\begin{abstract}
We investigate the interplay between strong electron correlations and charge-lattice interaction in cuprates. The coupling between half breathing bond stretching phonons and doped holes in the $t-t^{\prime}-J$ model is studied by limited phonon basis exact diagonalization method. Nonadiabatic electronphonon interaction leads to the splitting of the phonon spectral function at half-way to the zone boundary at $\vec{q}_{s}=\{( \pm \pi / 2,0),(0, \pm \pi / 2)\}$ and to low energy kink feature in the electron dispersion, in agreement with experimental observations. Another kink due to strong electron correlation effects is observed at higher energy, depending on the strength of the charge-lattice coupling.
\end{abstract}

PACS numbers: 71.10.Fd, 63.20.kk, 63.20.-e

There is a growing confidence that strong electronphonon interaction (EPI) manifests itself both in vibrational [1] and electronic [2] spectra of cuprates. The most puzzling feature of the cuprate phonon spectra is the anomaly of the half breathing bond stretching (HBBS) phonon occurring at half-way to the Brillouin zone (BZ) boundary in the [100]-direction, while the most debating feature of the electronic spectra are the kinks observed in Angle Resolved Photoemission Spectra (ARPES).

It was recently realized in the experimental community that it is highly important to measure both phonons spectra and ARPES on the same sample just to verify possible link between HBBS phonons and ARPES [3] . The results of the above studies support close connection between HBBS phonon anomaly and lowest energy kink in ARPES [3] and, thus, a model describing both anomalies within the same approach is strongly needed.

In the present Letter we study the low density limit (one hole on $4 \times 4$ lattice) of the extended $t-t^{\prime}-J$ model where holes are coupled to HBBS phonons [4]. In order to calculate the phonon spectral function (PSF) and hole spectral function (HSF), we generalize a recently introduced approach [5], based on limited phonon basis exact diagonalization (LPBED), without adopting selfconsistent Born (SCBA) and spin wave approximations [6, 7]. This method treats the non-adiabatic effects of the quantum phonon very effectively without approximations for the magnetic degrees of freedom. Because of exponential growth of the basis with size of the system, the $4 \times 4$ lattice has considerably denser quantum states than the smaller $\sqrt{10} \times \sqrt{10}$ system [8], so that it is possible to resolve fine structure of the PSF and HSF. For the first time we are able to reveal the shape of the PSF while previous studies [9 11] were restricted to at most the second moment of the response.

We show that EPI can lead to the splitting of the PSF at half-way to the BZ boundary in the [100] direction. We demonstrate that the splitting can be easily smeared out by a very small broadening and, thus, the mysterious evasive behavior of double-peak structure can be attributed to tiny variations of chemical composition, crystal quality and/or experimental setup. We argue that the splitting is a rather general phenomenon arising when the phonon branch interacts with a rather soft electronic excitation and we show that the HBBS phonon anomaly is linked to the lowest energy kink observed in the ARPES. Finally we emphasize that the same model supports the spectral kink at higher energy referred to colloquially as the waterfall [12].

McQueeney et al. [13] observed anomalous lineshape of HBBS phonons around $\mathbf{q}=(\pi / 2,0,0)$ and reported its strong temperature dependence. Subsequent studies confirmed that the HBBS phonon anomaly is due to the coupling of the HBBS phonons to the doped carriers. Indeed, the anomaly is absent in undoped compounds 1] and the hardening of the phonon spectra with heating [13, 14] excludes such sources of anomaly as anharmonicity or structural inhomogeneity. It is also agreed that HBBS phonon anomaly is located around $\mathbf{q}=(\pi / 2,0,0)$ at any doping. On the other hand, there is a controversy on the relation between the HBBS phonon anomaly and the lowest energy kink in the ARPES. This relation is often denied although the measurements of the phonon spectra and ARPES on the same sample of $\mathrm{Bi}_{2} \mathrm{Sr}_{1.6} \mathrm{La}_{0.4} \mathrm{Cu}_{2} \mathrm{O}_{6+x}$ suggest that the softening of the HBBS phonon mode matches the energy and momentum of this kink [3]. Another much debated question is the interpretation of the PSF structure at HBBS phonon anomaly. Actually the phonon peaks at $\vec{q}_{s}$ are poorly defined and two scenarios are possible. According to the single-branch interpretation (SBI) there is softening and a very large intrinsic linewidth of a single peak near $\mathbf{q}=(\pi / 2,0,0)$. On the contrary, the double-branch interpretation (DBI) implies the presence of two peaks: the first one is lo- 
cated at the unrenormalized energy and the second one is broad and soft. The pioneering study in Ref. 13] was followed by 17] whose results supported SBI. Next studies of similar material $\mathrm{La}_{1.875} \mathrm{Ba}_{0.125} \mathrm{CuO}_{4}$ led authors to conclusion about relevance of DBI [15]. Likewise, measurement of HBBS in $\mathrm{YBa}_{2} \mathrm{Cu}_{3} \mathrm{O}_{6+x}$ was interpreted in the framework of DBI 18]. Recently a "contamination hypothesis" [19] has been suggested, claiming that the SBI is correct because the inelastic neutron scattering measurement picks up the intensity from the k-vicinity $(\pi / 2, k, 0)$ of $\mathbf{q}=(\pi / 2,0,0)$ point and, thus, the "normal" component comes from transverse phonons. However, the above reasoning contradicts the results obtained on $\mathrm{La}_{2-x} \mathrm{Ba}_{x} \mathrm{CuO}_{4+\delta}$ at $x=0.14 \pm 0.01$ [20] by inelastic $\mathrm{x}$-ray scattering technique which has higher momentum resolution than that in neutron scattering measurements. "Contamination hypothesis" implies that the "normal" component must disappear by improving momentum resolution. Instead, the results of Ref. [20] support DBI. One can guess that the SBI vs DBI depends on the chemical composition [19], Ba-doped [15, 20] vs Sr-doped [14, 17] compound. Besides, since the softening is very sensitive to the broadening caused by decay channels and/or experimental resolution, SBI vs DBI must be strongly dependent on the compound, sample quality, and experimental setup.

We will show that many of the questions discussed above can be understood within the $t-t^{\prime}-J$ model including the coupling with HBBS. On the contrary density functional theory calculations 21, 22] do not predict any HBBS phonon anomaly [23]. Other approaches, especially those associating the anomaly with stripes, have difficulty with the position of HBBS phonon anomaly. Scenario suggested in Ref. 24] relates the HBBS softening to the Kohn anomaly at double Fermi momentum $2 k_{F}$ along the Fermi surface of stripes 24]. Here, in contrast to experiment, the softening must be $\theta$-independent. In a different theoretical proposal, HBBS anomaly is associated with stripe mediated collective charge excitations [25] or incommensurate low energy spin-fluctuations 26]. Within these scenarios, in contrast with experiment [14, 15, 20, 27, 28], wave vector of phonon anomaly strongly depends on doping level. Finally we note that phonon softening [9, 10], broadening [9], and correct position of the anomaly [11] have already been qualitatively explained by the coupling between phonons and density response of the $t-J$ model although none of those studies considered the shape of the phonon spectral function.

The Hamiltonian of the $t-t^{\prime}-J$-Holstein model in $2 \mathrm{D}$ is the sum of the electronic part and hole-phonon coupling Hamiltonian

$$
\begin{aligned}
H_{t t^{\prime} J}= & -t \sum_{i, \delta, \sigma} c_{i+\delta, \sigma}^{\dagger} c_{i, \sigma}-t^{\prime} \sum_{i, \delta^{\prime}, \sigma} c_{i+\delta^{\prime}, \sigma}^{\dagger} c_{i, \sigma} \\
& +\frac{J}{2} \sum_{i, \delta} S_{i+\delta} S_{i}-\frac{J}{8} \sum_{i, \delta} n_{i+\delta} n_{i}
\end{aligned}
$$

$H^{\prime}=\omega_{0} \sum_{q, \mu} a_{q, \mu}^{\dagger} a_{q, \mu}+\sum_{i, q, \mu}\left(M_{q, \mu} e^{i \vec{q} \cdot \vec{R}_{i}}\left(1-n_{i}\right) a_{q, \mu}+H . c.\right)$.

Here $J$ is the exchange interaction constant of the spinspin interaction, $t$ and $t^{\prime}$ are hopping amplitudes to nearest and next nearest neighbors. At site i ( $\mathrm{Cu}$ atoms), $S_{i}$ is the $\frac{1}{2}$-spin operator, $c_{i, \sigma}$ is the fermionic operators in the space without double occupancy, and $n_{i}$ is the number operator. $a_{q, \mu}$ is the phonon annihilation operator with momentum $q$ and $\mu=x$ or $y$ indicates the longitudinal polarization of the oxygen vibrations along the direction of the nearest neighbor $\mathrm{Cu}$ atoms (Oxygen atoms are located at $\vec{R}_{i}+a / 2 \mu, a$ being the lattice parameter). $M_{q, \mu}$ is the matrix element of the EPI: $M_{q, \mu}=g \omega_{0} / \sqrt{N} 2 i \sin \left(q_{\mu} / 2\right)$ where $N$ is the number of lattice sites and $\omega_{0}$ is the frequency of dispersionless optical phonon. The strength of EPI is characterized by dimensionless coupling constant $\lambda=\sum_{\vec{q}, \mu}\left|M_{q, \mu}\right|^{2} / 4 \omega_{0} t$. We chose parameters which correspond to hole doped cuprates: $J=0.4 t, t^{\prime}=-0.25 t, \omega_{0}=0.15 t$, and set $t=a=\hbar=1$. The PSF is expressed as

$$
D(\vec{q}, \omega+i \eta)=-\frac{1}{\pi} \Im \frac{1}{D_{0}^{-1}(\vec{q}, \omega+i \eta)-\Sigma(\vec{q}, \omega+i \eta)},
$$

where the self-energy $\Sigma(\vec{q}, \omega+i \eta)$ is given by

$$
\Sigma(\vec{q}, \omega+i \eta)=\frac{\left|M_{q, x}\right|^{2} \Pi(\vec{q}, \omega)}{\left(1+\left|M_{q, x}\right|^{2} \Pi(\vec{q}, \omega) / D_{0}^{-1}(\vec{q}, \omega+i \eta)\right)} .
$$

Here $D_{0}(\vec{q}, \omega+i \eta)$ is the bare phonon Green function and $\Pi(\vec{q}, \omega)=P(\vec{q}, \omega+i \eta)+P(\vec{q},-\omega-i \eta)$ is the polarization insertion with

$$
P(\vec{q}, \omega+i \eta)=\left\langle\psi_{0}\left|O^{\dagger} \frac{1}{\omega+i \eta-H+E_{0}} O\right| \psi_{0}\right\rangle .
$$

We choose the ground state (GS) $\left|\psi_{0}\right\rangle$ as a linear superposition with equal weights of the 4 degenerate states corresponding to $\vec{k}=\left( \pm \frac{\pi}{2}, \pm \frac{\pi}{2}\right)$ with energy $E_{0}, \eta$ is a broadening factor that shifts the poles of $D(\vec{q}, \omega)$ in the complex plane, and $O=\sum_{i} e^{i \vec{q} \cdot \vec{R}_{i}}\left(1-n_{i}\right)$. The increase of broadening factor $\eta$ has the physical meaning of phonon damping or limited experimental resolution.

The ground state $\left|\psi_{G S}\right\rangle$ and the function $P(\vec{q}, \omega+i \eta)$ are obtained by modified 29] and standard Lanczos methods, respectively, within the LPBED method [5] introduced for the $t-t^{\prime}-J$-Holstein model.

In Fig. 1 we plot the PSF $D(\vec{q}, \omega)$ for different wavevectors along the $(1,0)$ direction in the BZ. For the chosen values of the model parameters the system undergoes, in agreement with [30], a crossover towards strong EPI regime for $\lambda_{c} \simeq 0.5$. The anomalous softening of the phonon mode at $\vec{q}_{s}=(\pi / 2,0)$ is already observed at moderate values $\lambda<\lambda_{c}$ of the hole-phonon coupling $(\lambda=0.3)$. We stress that for a large value of broadening factor $\eta=0.08$ the phonon peak softens and broadens at $\vec{q}_{s}$ (Fig. 1a), supporting, thus, SBI. On the other hand, 


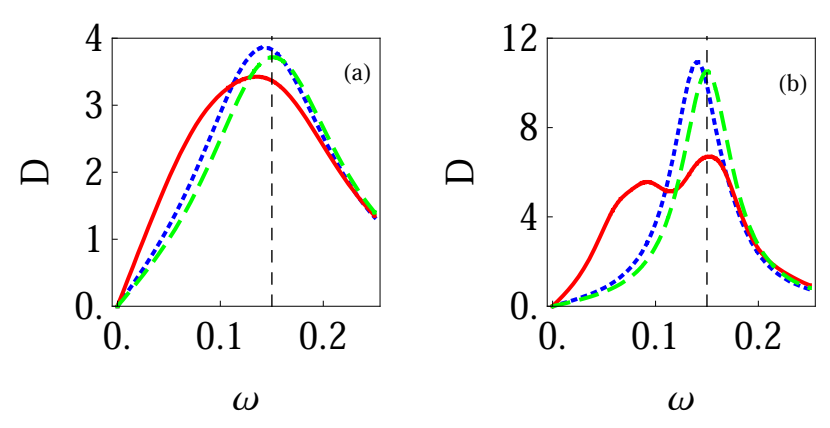

FIG. 1: (Color online) (a) The PSF for $\lambda=0.3$ at three different values of $\vec{q}(\vec{q}=(0,0)$ (dashed green line), $\vec{q}=(\pi / 2,0)$ (solid red line), and $\vec{q}=(\pi, 0)$ (dotted blue line)) with $\eta=0.08$; (b) the PSF with a smaller broadening factor $\eta$, $\eta=0.03$. The vertical lines indicate the bare phonon frequency $\omega_{0}$.

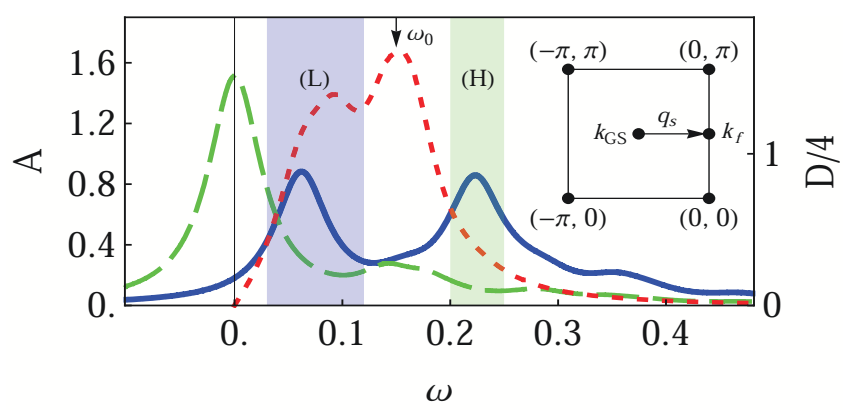

FIG. 2: (Color online) HSF $A(\vec{k}, \omega)$, for $\lambda=0.3$ at two different values of $\vec{k}(\vec{k}=(\pi / 2, \pi / 2)$ (dashed green line), $\vec{k}=(0, \pi / 2)$ (solid blue line)) with $\eta=0.03$. The energy is measured with respect to the GS energy. The phonon Green function at $\vec{q}=(\pi / 2,0)$ (with the same value of $\eta$ ) is plotted too for comparison (red dotted line). In the inset sketch of one of transitions involved in the PSF calculation.

reducing the broadening factor to $\eta=0.03$ two-peak structure turns out(Fig. 1b), that is in agreement with the experimental observations reported in Ref. 15, 20].

To clarify the physical nature of the splitting, we study the hole spectral weight function $A(\vec{k}, \omega)$ at the wavevectors $\vec{k}_{f}$ that are reached starting from GS wavenumber at $( \pm \pi / 2, \pm \pi / 2)$ through phonon momentum $\vec{q}_{s}=(\pi / 2,0)$. For example, as shown in Fig.2, by starting from $\vec{k}=$ $(-\pi / 2, \pi / 2)$ we get $\vec{k}_{f}=(0, \pi / 2)$ after absorbing (emitting) a phonon with momentum $-\vec{q}_{s}\left(\vec{q}_{s}\right)$. The lowest energy peak in $D\left(\vec{q}_{s}, \omega\right)$ (dotted red curve in Fig. 2) is close to the lowest energy peak of $A\left(\vec{k}_{f}, \omega\right)$ (solid blue curve in Fig. 2). Measuring energy from the GS, denoted by vertical line in Fig. 2, one can see that the lowest peaks in phonon and hole spectral functions are softer than the phonon energy $\omega_{0}=0.15 \mathrm{t}$. We note that the significant renormalization of the hole spectral weight, with respect to GS one, indicates strong coupling between phonon at $\vec{q}_{s}$ and hole at $\vec{k}_{f}$. The high energy peak in $D\left(\vec{q}_{s}, \omega\right)$ is located at energy close to $\omega_{0}$, and no peak at the same energy is found in $A(\vec{k}, \omega)$. It is shown below that it is due to the phononic nature of the high energy resonance of the PSF.

To give a simple explanation of the above scenario one has to realize that the excited electronic state $|\psi\rangle_{(0, \pi / 2)}^{0}|0\rangle$ with the momentum $(0, \pi / 2)$ and without phonons is linked by the matrix elements of EPI with a group of 8 degenerate states $|\psi\rangle_{( \pm \pi / 2, \pm \pi / 2)}^{0} a_{q, \mu}^{\dagger}|0\rangle$ where the electronic subsystem is in the GS and one phonon is excited. The momentum conservation $( \pm \pi / 2, \pm \pi / 2)+$ $\vec{q}=(0, \pi / 2)$ determines the phonon momenta $\vec{q}$. The energies of the electronic subsystem at corresponding momenta are $\epsilon^{0}(0, \pi / 2)$ and $\epsilon^{0}(\pi / 2, \pi / 2)$, respectively. Then, choosing a set of the model parameters appropriate for cuprates, the resonance relation $\epsilon^{0}(0, \pi / 2) \approx$ $\epsilon^{0}(\pi / 2, \pi / 2)+\omega_{0}$ is satisfied. Hence, even small matrix elements induce strong effects both in electronic and vibrational subsystems. Analytic diagonalization of this degenerate $9 \times 9$ matrix gives 9 levels. The lowest state $L$ has energy below $\omega_{0}$ and has components on both onephonon and zero-phonon states. This state corresponds to the peaks of electronic $A(\vec{k}=(0, \pi / 2), \omega)$ and bosonic $D(\vec{q}=(\pi / 2,0), \omega)$ spectral functions at $\omega<0.1$ (see the area around letter $L$ in Fig. 2). In the highest energy range, (see the area around letter $H$ in in Fig. 2), both spectral functions collect contributions at $\omega \geq 0.2$. However, the $H$-state is of predominantly electronic origin with a large peak in the hole spectral function while it generates a weak structure in the PSF, hardly observable in the numerical data. The last 7 degenerate levels with energy $\epsilon^{0}(\pi / 2, \pi / 2)+\omega_{0}$ have no projection on the vacuum boson state: this explains why the hole spectral weight function does not show any peak at this energy, at a distance $\omega_{0}$ from GS energy. The energies of all levels of the above simple analytic solution are in qualitative agreement with that provided by the numeric LPBED method in Fig. 2. So, we conclude that the doubling of the phonon peak is due to coupling between holes and lattice, that lifts a degeneracy and produces one additional state with energy less than $\omega_{0}$.

Generically, the EPI involving electronic states with energies considerably larger than the phonon frequency does not lead to any splitting since the adiabatic approximation is valid and electron produces only a renormalization of the adiabatic potential [31]. On the other hand, when the electronic excitation is soft and its energy is comparable with phonon frequency, the nonadiabatic corrections play a crucial role 32 and one can observe exotic spectral functions as it is seen in Fig. 1b and in experiments on cuprates.

The clear connection, established by the above considerations, between phonon at $\vec{q}_{s}$ and hole at $\vec{k}_{f}$ encouraged us to search for an interplay between the HBBS phonon anomaly and the lowest energy kink in the dispersion of quasiparticles in cuprates [33]. In Fig.3a we plot hole dis- 

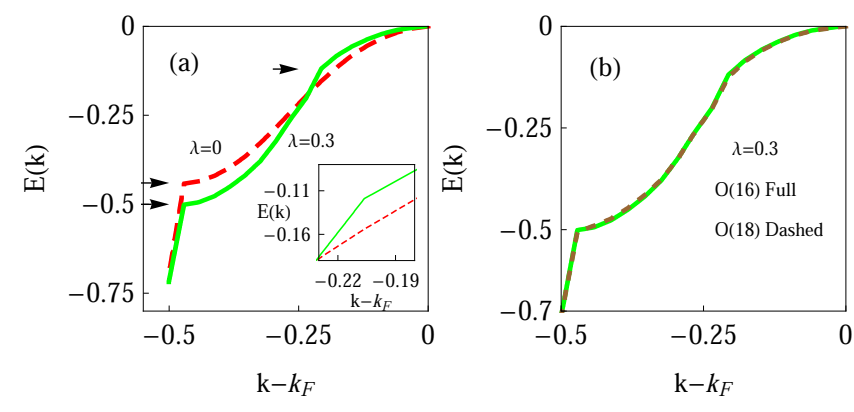

FIG. 3: (Color online) (a) Energy vs $\left(\vec{k}-\vec{k}_{F}\right) / \pi$ dispersion relations along nodal direction from $\vec{k}_{F}$ to $\Gamma$ point at $\lambda=0.3$ (solid) and $\lambda=0$ (dashed). Arrows mark kink positions. (b) Dispersion relation as a function of oxygen isotope exchange $\left({ }^{16} \mathrm{O}\right.$ (solid) and ${ }^{18} \mathrm{O}($ dashed $\left.)\right)$ at $\lambda=0.3$.

persion derived from momentum distribution curves, i.e. we find the $\vec{k}$ for which the hole Green function $A(\vec{k}, \omega)$ has maximum at fixed $\omega$ 34]. The energy, measured from GS, is plotted versus the wavenumber $\left(\vec{k}-\vec{k}_{F}\right)$, where $\vec{k}_{F}$ is the momentum corresponding to the minimum of the hole dispersion relation. We find that, when $E_{k}-E_{k_{F}}$ is about $\omega_{0}=0.15$, the curve exhibits a slope change related to the coupling between the bare phonon energy and the electronic band in the bare $t-t^{\prime}-J$ model. This kink does not appear in absence of the hole-phonon coupling. On the other hand, we obtain another kink at higher energy (see Fig.3a) [12]. This distinctive feature is related to the strong electron correlations since it is observed also in the bare $t-t^{\prime}-J$ model. At $\lambda=0$ the kink is located around the exchange interaction energy $J$, in agreement with results by Chakraborty et al. [35] within the Hubbard model. Furthermore, the kink is shifted at higher energy by increasing the hole-phonon coupling. We also investigated the effect of the oxygen isotope substitution [5] on ARPES. The results (Fig.3b) show a negligible (below 0.01t) shift upon oxygen isotope exchange, that is in agreement with recent experimental observations by Douglas et al. 36]. All these data point out that the scenario based on the interplay between strong electron correlations and hole-phonon interaction is able to capture many physical distinctive features of high temperature superconductors.

In conclusion, we showed that the EPI, in the presence of strong correlations, can lead to the splitting of the phonon spectral function at half-way to the BZ boundary in the [100] direction. We demonstrated that the splitting can be easily smeared out by very small broadening of the eigenstates. The same physical mechanism can explain both HBBS phonon anomaly and lowest energy kink in the ARPES. Finally we found that the isotope effect on ARPES is negligible in accordance with experiment. These results support the claim that strong electron correlations and charge lattice interaction are crucial in understanding cuprate experimental features.

Work supported by RFBR 07-02-0067a (A.S.M.), and Grant- in-Aids No. 15104006, No. 16076205, No. 17105002, No. 19048015, and NAREGI Japan (N.N.); G.D.F., V.C. and C.A.P. received financial support from MIUR-PRIN 2007 under Prot. No. 2207FW3MJX003.

[1] L. Pintschovius, Phys. Status Solidi b 242, 30 (2005)

[2] O. Gunnarsson and Rösch, J. Phys.: Condens. Matter 20, 043201 (2008)

[3] J. Graf et al., Phys. Rev. Lett. 100, 227002 (2008)

[4] N. Bulut and D. J. Scalapino, Phys. Rev. B 54, 14971 (1996); S. Ishihara and N. Nagaosa, Phys. Rev. B 69, 144520 (2004)

[5] G. De Filippis et al., Phys. Rev. B 80, 195104 (2009)

[6] C. L. Kane et al., Phys. Rev. B 39, 6880 (1989)

[7] Z. Liu and E. Manousakis, Phys. Rev. B 45, 2425 (1992)

[8] B. Bauml et al., Phys. Rev. B 58, 3663 (1998)

[9] G. Khaliullin and P. Horsch, Phys. Rev. B 54, R9600 (1996); Physica C 282-287, 1751 (1997)

[10] O. Rösch and O. Gunnarsson, Phys. Rev. Lett. 92, 146403 (2004); ibid 93, 237001 (2004)

[11] P. Horsch et al., Physica C 341-348, 117 (2000)

[12] J. Graf et al., Phys. Rev. Lett. 98, 067004 (2007)

[13] R. J. McQueeney et al., Phys. Rev. Lett. 82, 628 (1999)

[14] D. Reznik et al., J. Low. Temp. Phys. 147, 353 (2007)

[15] D. Reznik et al., Nature 440, 1170 (2006)

[16] L. Pintschovius et al., Phys. Rev. B 74, 174514 (2006)

[17] L. Pintschovius and M. Braden, Phys. Rev. B 60, R15039 (1999)

[18] F. Stercel et al., Phys. Rev. B 77, 014502 (2008)

[19] D. Reznik, Adv. Cond. Matt. Phys. 2010, 523549 (2010)

[20] M. d'Astuto et al., Phys. Rev. B 78, 140511(R) (2008)

[21] F. Giustino et al., Nature 452, 975 (2008)

[22] K. P. Bohnen, R. Heid and M. Krauss, Europhys. Lett. 64, 104 (2003)

[23] D. Reznik et al., Nature 455, E6 (2008)

[24] S.I. Mukhin et al., Phys. Rev. B 76, 174521 (2007); R. Citro et al., Eur. Phys. J. B 63, 179-185 (2008)

[25] E. Kaneshita et al., Phys. Rev. Lett. 88, 115501 (2002)

[26] S. A. Kivelson et al., Rev. Mod. Phys. 75, 1201 (2003)

[27] J. Graf et al., Phys. Rev. B 76, 172507 (2007)

[28] H. Uchiyama et al., Phys. Rev. Lett. 92, 197005 (2004)

[29] E. Dagotto and A. Moreo, Phys. Rev. D 31, 865 (1985)

[30] A. S. Mishchenko and N. Nagaosa, Phys. Rev. Lett. 93, 036402 (2004); Phys. Rev. B 73, 092502 (2006); A. S. Mishchenko et al, Phys. Rev. Lett. 100, 166401 (2008)

[31] M. Born and R. Oppenheimer, Ann. d. Phys. 84, 457 (1927)

[32] K. A. Kikoin and A. S. Mishchenko, Zh. Eksp. Teor. Fiz. 104, 3810 (1993) [JETP 77, 828 (1993)]

[33] A. Lanzara et al., Nature 412, 510 (2001)

[34] The full $\vec{k}$ dependence is facilitated by twisted boundary conditions (see T. Xiang and J. M. Wheatley, Phys. Rev. B 54, R12653 (1996))

[35] S. Chakraborty et al., Phys. Rev. B 78, 212504 (2008)

[36] J. F. Douglas et al., Nature 446, 05738 (2007) 